\title{
An Iterative Algorithm for Generalized Mixed Equilibrium Problems and Fixed Points of Nonexpansive Semigroups
}

\author{
Jong Kyu Kim¹, Salahuddin², Won Hee Lim¹ \\ ${ }^{1}$ Department of Mathemarics Education, Kyungnam University Changwon, Gyeongnam, Korea \\ ${ }^{2}$ Department of Mathematics, Jazan University, Jazan, Kingdom of Saudi Arabia \\ Email: jongkyuk@kyungnam.ac.kr, salahuddin12@mailcity.com, worry@kyungnam.ac.kr
}

How to cite this paper: Kim, J.K., Salahuddin and Lim, W.H. (2017) An Iterative Algorithm for Generalized Mixed Equilibrium Problems and Fixed Points of Nonexpansive Semigroups. Journal of Applied Mathematics and Physics, 5, 276-293. https://doi.org/10.4236/jamp.2017.52025

Received: January 3, 2017

Accepted: February 12, 2017

Published: February 15, 2017

\begin{abstract}
In this works, by using the modified viscosity approximation method associated with Meir-Keeler contractions, we proved the convergence theorem for solving the fixed point problem of a nonexpansive semigroup and generalized mixed equilibrium problems in Hilbert spaces.
\end{abstract}

\section{Keywords}

Meir-Keeler Contraction Mappings, Left Regular, Generalized Mixed Equilibrium Problems, Variational Inequalities, $\alpha$-Inverse Strongly Monotone Mappings, Nonexpansive Semigroups

\section{Introduction}

As you know, there are many problems that are reduced to find solutions of equilibrium problems which cover variational inequalities, fixed point problems, saddle point problems, complementarity problems as special cases. Equilibrium problem which was first introduced by Blum and Oettli [1] has been extensively studied as effective and powerful tools for a wide class of real world problems, which arises in economics, finance, image reconstruction, ecology, transportation network and related optimization problems.

From now on, we assume that $H$ is a real Hilbert space with inner product $\langle\cdot, \cdot\rangle$ and norm $\|\cdot\|$, and $K$ is a nonempty closed convex subset of $H . \mathbb{R}$ is denoted by the set of real numbers. Let $G: K \times K \rightarrow \mathbb{R}$ be a bifunction. Blum and Oettli [1] consider the equilibrium problem of finding $x \in K$ such that

$$
G(x, y) \geq 0, \forall y \in K \text {. }
$$

The solution set of problem (1.1) is denoted by $E P(G)$, i.e., 


$$
E P(G)=\{x \in K: G(x, y) \geq 0, \forall y \in K\}
$$

Recently the so-called generalized mixed equilibrium problem has been investigated by many authors [2] [3]. The generalized mixed equilibrium problem is to find $x \in K$. such that

$$
G(x, y)+\varphi(y)+\langle A x, y-x\rangle \geq \varphi(x), \forall y \in K,
$$

where $A: K \rightarrow H$ is a mapping and $\varphi: K \rightarrow \mathbb{R} \cup\{+\infty\}$ is a real valued function. We use $\operatorname{GMEP}(G, A, \varphi)$ to denote the solution set of generalized mixed equilibrium problem i.e.,

$$
\operatorname{GMEP}(G, A, \varphi)=\{x \in K: G(x, y)+\langle A x, y-x\rangle+\varphi(y)-\varphi(x) \geq 0, \forall y \in K\} .
$$

The problem (1.2) is very general in the sense that it includes, as special cases, optimization problems, variational inequality problem, minimax problems, the Nash equilibrium problems in noncooperative games and others (see [4] [5] [6] [7] [8] [9] [10] [11] [12]).

Special Cases: The following problems are the special cases of problem (1.2).

1) If $A=0$ then (1.2) is equivalent to finding $x \in K$ such that

$$
G(x, y)+\varphi(y)-\varphi(x) \geq 0, \forall y \in K,
$$

is called mixed equilibrium problems.

2) If $G=0$ then (1.2) is equivalent to finding $x \in K$ such that

$$
\langle A x, y-x\rangle+\varphi(y)-\varphi(x) \geq 0, \forall y \in K,
$$

is called mixed variational inequality of Browder type [13].

3) If $\varphi=0$ then (1.2) is equivalent to find $x \in K$ such that

$$
G(x, y)+\langle A x, y-x\rangle \geq 0, \forall y \in K,
$$

is called generalized equilibrium problems (shortly, (GEP)). We denote GEP(G,A) the solution set of problem (GEP).

4) If $A=0$ and $\varphi=0$ then (1.2) is equivalent to (1.1).

5) Let $G(x, y)=\langle A x, y-x\rangle$, for all $x, y \in K$. Then we see that (1.1) is reduces to the following classical variational inequalities for finding $x \in K$ such that

$$
\langle A x, y-x\rangle \geq 0, \forall y \in K .
$$

It is known that $x \in K$ is a solution to (1.6) if and only if $x$ is a fixed point of the mapping $P_{K}(I-\rho A)$, where $\rho>0$ is a constant and $I$ is an identity mapping.

Let $T: K \rightarrow K$ be a mapping from $K$ into itself. Let denote $F(T)$ the set of fixed points of the mapping T. A mapping $T$ is said to be nonexpansive if

$$
\|T x-T y\| \leq\|x-y\|, \forall x, y \in K .
$$

A mapping $T$ is said to be contractive if there exists a constant $\alpha \in[0,1)$ such that

$$
\|T x-T y\| \leq \alpha\|x-y\|, \forall x, y \in K .
$$

A mapping $T$ is called $\alpha$-inverse strongly monotone if there exists a constant $\alpha>0$ such that 


$$
\langle T x-T y, x-y\rangle \geq \alpha\|T x-T y\|^{2}, \forall x, y \in K .
$$

Remark 1.1 Every $\alpha$-inverse strongly monotone mapping is monotone and $\frac{1}{\alpha}$-Lipschitz continuous.

In 1967, Halpern [14] introduced the following iterative method for a nonexpansive mapping $T: K \rightarrow K$ in a real Hilbert space, for finding $x_{1} \in K$ and

$$
x_{n+1}=\alpha_{n} u+\left(1-\alpha_{n}\right) T x_{n}, n \geq 1
$$

where $\left\{\alpha_{n}\right\} \subset(0,1)$ and $u \in K$ is fixed.

Moudafi [15] introduced the viscosity approximation method for a nonexpansive mapping $T$ as follows: For finding $x_{1} \in K$ and

$$
x_{n+1}=\alpha_{n} f\left(x_{n}\right)+\left(1-\alpha_{n}\right) T x_{n}, n \geq 1
$$

where $\left\{\alpha_{n}\right\} \subset(0,1)$ and $f$ is a contraction mapping.

A viscosity approximation method with Meir-Keeler contraction was first studied by Suzuki [16]. Very recently Petrusel and Yao [17] studied the following viscosity approximation method with a generalized contraction: for finding $x_{0} \in K$ and

$$
x_{n+1}=\alpha_{n+1} f\left(x_{n}\right)+\left(1-\alpha_{n+1}\right) T_{n+1} x_{n}, n \geq 0,
$$

where $\left\{\alpha_{n}\right\} \subset(0,1)$ and $\left\{T_{n}\right\}_{n=1}^{\infty}$ is a family of nonexpansive mappings on $K$.

Takahashi and Takahashi [18] introduced the following iterative scheme for solving a generalized equilibrium problems and a fixed point problems of a nonexpansive mapping $T$ in a Hilbert spaces $H$ : Finding $x_{1}, u \in K$ and

$$
\left\{\begin{array}{l}
u_{n} \in K \text { such that } \\
G\left(u_{n}, y\right)+\left\langle A x_{n}, y-u_{n}\right\rangle+\frac{1}{r_{n}}\left\langle y-u_{n}, u_{n}-x_{n}\right\rangle \geq 0, y \in K, \\
x_{n+1}=\beta_{n} x_{n}+\left(1-\beta_{n}\right) T\left[\alpha_{n} u+\left(1-\alpha_{n}\right) u_{n}\right], n \geq 1,
\end{array}\right.
$$

where $\left\{\alpha_{n}\right\} \subset(0,1),\left\{\beta_{n}\right\} \subset(0,1),\left\{r_{n}\right\} \subset(0, \infty)$ and $A$ is an $\alpha$-inverse strongly monotone mapping. They proved that the sequence $\left\{x_{n}\right\}$ generated by (1.9) strongly converges to an element in $F(T) \cap G E P(G, A)$ under suitable conditions.

In this paper, from the recent works [19] [20] [21] [22] [23] [24] [25] [26], we introduced an iterative scheme by the modified viscosity approximation method associated with Meir-Keeler contraction (see [27]) for solving the generalized mixed equilibrium problems and fixed point problem of a nonexpansive semigroup in Hilbert spaces, and also we discussed a convergence theorem. Finally we apply our main results for commutative nonexpansive mappings and semigroup of strongly continuous mappings.

\section{Preliminaries}

Let $S$ be a semigroup and $\ell^{\infty}(S)$ be the Banach space of all bounded real valued functionals on $S$ with superimum norm. For each $s \in S$, we define the left and right translation operators $l_{s}$ and $r_{s}$ on $\ell^{\infty}(S)$ by $\left(l_{s} f\right)(t)=f(s t)$ 
and $\left(r_{s} f\right)(t)=f(t s)$ for each $t \in S$ and $f \in \ell^{\infty}(S)$, respectively. Let $X$ be a subspace of $\ell^{\infty}(s)$ containing 1 . An element $\mu$ in the dual space $X^{*}$ of $X$ is said to be a mean on $X$ if $\|\mu\|=\mu(1)=1$. We denote the value of $\mu$ at the function $f$ by $\mu(f)$. According to the time and circumstances, we write the value $\mu(f)$ by $\mu_{t}(f(t))$ or $\int f(t) d \mu(t)$. It is well known that $\mu$ is a mean of $X$ if and only if for each $f \in X$,

$$
\inf _{s \in S} f(s) \leq \mu(f) \leq \sup _{s \in S} f(s) .
$$

Let $X$ be a translation invariant subspace of $\ell^{\infty}(S)$ (i.e., $l_{s} X \subset X$ and $r_{s} X \subset X$ for each $s \in S$ ) containing 1 . Then a mean $\mu$ on $X$ is said to be left invariant (resp. right invariant) if $\mu\left(l_{s} f\right)=\mu(f) \quad$ (resp. $\left.\mu\left(r_{s} f\right)=\mu(f)\right)$ for each $s \in S$ and $f \in X$. A mean $\mu$ on $X$ is said to be invariant if $\mu$ is both left and right invariant [28] [29]. $S$ is said to be left (resp. right) amenable if $X$ has a left (resp. right) invariant mean. $S$ is amenable if $S$ is left and right amenable [30]. In this case $\ell^{\infty}(S)$ also has an invariant mean. It is known that $\ell^{\infty}(S)$ is amenable when $S$ is commutative semigroup or solvable group. However the free group or semigroup of two generators is not left or right amenable (see [31]). A net $\left\{\mu_{\alpha}\right\}$ of mean on $X$ is said to be left regular if

$$
\lim _{\alpha}\left\|l_{s}^{*} \mu_{\alpha}-\mu_{\alpha}\right\|=0
$$

for each $s \in S$, where $l_{s}^{*}$ is the adjoint operator of $l_{s}$.

Let $K$ be a nonempty closed convex subset of $H$. A family $\mathcal{S}=\{T(s): s \in S\}$ is called a nonexpansive semigroup on $S$ if for each $s \in S$, the mapping $T(s): K \rightarrow K$ is nonexpansive and $T(s t)=T(s) T(t)$ for each $s, t \in S$ (see [30] [30]). We denote by $F(\mathcal{S})$ the set of common fixed point of $S$, i.e.,

$$
F(\mathcal{S})=\bigcap_{s \in S} F(T(s))=\bigcap_{s \in S}\{x \in K: T(s) x=x\} .
$$

Assume that $B_{r}$ is a open ball of radius $r$ centered at 0 and $\overline{c o} A$ is a closed convex hull of $A \subset H$. For $\epsilon>0$ and a mapping $T: D \rightarrow H$, the set of $\epsilon$-approximate fixed points of $T$ will be denoted by $F_{\epsilon}(T, D)$, i.e.,

$$
F_{\epsilon}(T, D)=\{x \in D:\|x-T x\| \leq \epsilon\} .
$$

Lemma 2.1 [32] Let $f$ be a function of a semigroup $S$ into a Banach space $E$ such that the weak closure of $\{f(t): t \in S\}$ is weakly compact and $X$ a subspace of $\ell^{\infty}(S)$ containing all the function $t \rightarrow\left\langle f(t), x^{*}\right\rangle$ with $x^{*} \in E^{*}$. Then for any $\mu \in X^{*}$ there exists a unique element $f_{\mu}$ in $E$ such that for all $x^{*} \in E^{*}$,

$$
\left\langle f_{\mu}, x^{*}\right\rangle=\mu_{t}\left\langle f(t), x^{*}\right\rangle
$$

Moreover if $\mu$ is a mean on $X$ then

$$
\int f(t) d \mu(t) \in \overline{c o}\{f(t): t \in S\}
$$

We can write $f_{\mu}$ by $\int f(t) d \mu(t)$.

Lemma 2.2 [32] Let $K$ be a closed convex subset of a Hilbert space H.Let 
$\mathcal{S}=\{T(s): s \in S\}$ be a nonexpansive semigroup from $K$ into itself such that $F(\mathcal{S}) \neq \varnothing, X$ be a subspace of $\ell^{\infty}(S)$ containing 1, the mapping

$t \rightarrow\langle T(t) x, y\rangle$ be an element of $X$ for each $x \in K$ and $y \in H$ and $\mu$ be a mean on $X$. If we write $T_{\mu} x$ instead of $\epsilon T(t) x d \mu(t)$, then the following statements hold:

1) $T_{\mu}$ is a nonexpansive mapping from $K$ into $K$,

2) $T_{\mu} x=x$ for each $x \in F(\mathcal{S})$,

3) $T_{\mu} x \in \overline{\operatorname{co}}\{T(t) x: t \in S\}$, for each $x \in K$;

4) if $\mu$ is left invariant then $T_{\mu}$ is a nonexpansive retraction from $K$ into $F(\mathcal{S})$.

Let $K$ be a nonempty closed convex subset of a real Hilbert space $H$. Then for any $x \in H$ there exists a unique nearest point in $K$, denoted by $P_{K}(x)$ such that for all $y \in K$,

$$
\left\|x-P_{K}(x)\right\| \leq\|x-y\|,
$$

where $P_{K}$ is the metric projection of $H$ onto $K$. We also know that for $x \in H$ and $z \in K, z=P_{K} x$ if and only if for all $y \in K$,

$$
\langle x-z, y-z\rangle \leq 0 \text {. }
$$

A mapping $\psi: \mathbb{R}_{+} \rightarrow \mathbb{R}_{+}$is said to be an $L$-function if $\psi(0)=0, \psi(t)>0$ for each $t>0$ and for every $s>0$ there exists $u>s$ such that $\psi(t) \leq s$ for all $t \in[s, u]$. As a consequence, every $L$-function $\psi$ satisfies $\psi(t)<t$ for each $t>0$.

Definition 2.3 Let $(X, d)$ be a metric space. A mapping $f: X \rightarrow X$ is said to be a

1) $(\psi, L)$-contraction if $\psi: \mathbb{R}_{+} \rightarrow \mathbb{R}_{+}$is an $L$-function and

$$
d(f(x), f(y))<\psi(d(x, y))
$$

for all $x, y \in X$ with $x \neq y$;

2) Meir-Keeler type mapping if for each $\epsilon>0$ there exists $\delta=\delta(\epsilon)>0$ such that for each $x, y \in X$ with $d(x, y)<\epsilon+\delta$ we have $d(f(x), f(y))<\epsilon$ (see [33] [34]).

Theorem 2.4 [34] Let $(X, d)$ be a complete metric space and $f: X \rightarrow X$ is a Meir-Keeler type mapping. Then $f$ has a unique fixed point.

Theorem 2.5 [35] Let $(X, d)$ be a complete metric space and $f: X \rightarrow X$ is a mapping. Then the following statements are equivalent.

1) $f$ is a Meir-Keeler type mapping;

2) there exists an $L$-function $\psi: \mathbb{R}_{+} \rightarrow \mathbb{R}_{+}$such that $f$ is a $(\psi, L)$-contraction.

Theorem 2.6 [16] Let $K$ be a convex subset of a Banach space $E$ and let $f: K \rightarrow K$ be a Meir-Keeler type mapping. Then for each $\epsilon>0$ there exists $r \in(0,1)$ such that for each $x, y \in K$ with $\|x-y\| \geq \epsilon$ we have

$$
\|f(x)-f(y)\| \leq r\|x-y\| .
$$

Proposition 2.7 [31] Let $K$ be a convex subset of a Banach space E, $T$ be a nonexpansive mapping on $K$ and $f: K \rightarrow K$ be a Meir-Keeler type 
mapping. Then the following statements hold:

1) $T \circ f$ is a Meir-Keeler type mapping on $K$.

2) For each $\alpha \in(0,1)$, the mapping $x \rightarrow \alpha f(x)+(1-\alpha) T(x)$ is a MeirKeeler type mapping on $K$.

Lemma 2.8 [36] Assume that $\left\{a_{n}\right\}$ is a sequence of nonnegative real number such that

$$
a_{n+1} \leq\left(1-\rho_{n}\right) a_{n}+\rho_{n} \delta_{n}, n \geq 1,
$$

where $\left\{\rho_{n}\right\}$ is a sequence in $(0,1)$ and $\left\{\delta_{n}\right\}$ is a sequence in $\mathbb{R}$ satisfying

1) $\sum_{n=1}^{\infty} \rho_{n}=\infty$;

2) $\limsup _{n \rightarrow \infty} \delta_{n} \leq 0$ or $\sum_{n=1}^{\infty}\left|\rho_{n} \delta_{n}\right|<\infty$.

Then $\lim _{n \rightarrow \infty} a_{n}=0$.

Lemma 2.9 [37] Let $\left\{x_{n}\right\}$ and $\left\{z_{n}\right\}$ be bounded sequences in a Banach space E such that

$$
x_{n+1}=\left(1-\beta_{n}\right) z_{n}+\beta_{n} x_{n}, \forall n \geq 1,
$$

where $\left\{\beta_{n}\right\}$ is a real sequence in $(0,1)$ with

$$
0<\liminf _{n \rightarrow \infty} \beta_{n} \leq \limsup _{n \rightarrow \infty} \beta_{n}<1 .
$$

If

$$
\limsup _{n \rightarrow \infty}\left(\left\|z_{n+1}-z_{n}\right\|-\left\|x_{n+1}-x_{n}\right\|\right) \leq 0
$$

then

$$
\lim _{n \rightarrow \infty}\left\|z_{n}-x_{n}\right\|=0
$$

Lemma 2.10 [38] Let $0<p \leq t_{n} \leq q<1$ for all $n \geq 1$. Suppose that $\left\{x_{n}\right\}$ and $\left\{y_{n}\right\}$ are sequences in $H$ such that

$$
\underset{n \rightarrow \infty}{\limsup }\left\|x_{n}\right\| \leq r, \quad \limsup _{n \rightarrow \infty}\left\|y_{n}\right\| \leq r
$$

and

$$
\lim _{n \rightarrow \infty}\left\|t_{n} x_{n}+\left(1-t_{n}\right) y_{n}\right\|=r
$$

for some $r \geq 0$. Then we have

$$
\lim _{n \rightarrow \infty}\left\|x_{n}-y_{n}\right\|=0
$$

Lemma 2.11 [39] Let $K$ be a nonempty closed convex subset of a real Hilbert space $H$ and $T: K \rightarrow K$ be a nonexpansive mapping with $F(T) \neq \varnothing$. Then $I-T$ is demiclosed at zero, that is, for all sequence $\left\{x_{n}\right\} \subset K$ with $x_{n} \rightarrow y$ and $\left\|x_{n}-T x_{n}\right\| \rightarrow 0$ it follows that $y=T y$.

For solving the equilibrium problem we assume that bifunction $G$ satisfies the following conditions:

(A1) $G(x, x)=0, \forall x \in K$;

(A2) $G$ is monotone, i.e., $G(x, y)+G(y, x) \leq 0, \forall x, y \in K$;

(A3) for each $x, y, z \in K, \lim _{t \rightarrow 0} G(t z+(1-t) x, y) \leq G(x, y)$;

(A4) for each $x \in K, y \rightarrow G(x, y)$ is convex and lower semicontinuous.

Lemma 2.12 [1] Let $K$ be a nonempty closed convex subset of a real Hilbert 
space $H$ and $G$ be a bifunction from $K \times K$ to $\mathbb{R}$ satisfying $(A 1)-(A 4)$. Then for any $r>0$ and $x \in H$, there exists $z \in K$ such that

$$
G(z, y)+\frac{1}{r}\langle y-z, z-x\rangle \geq 0, \forall y \in K \text {. }
$$

Further, if

$$
T_{r} x=\left\{z \in K: G(z, y)+\frac{1}{r}\langle y-z, z-x\rangle \geq 0, \forall y \in K\right\},
$$

then we have the followings:

1) $T_{r}$ is single-valued;

2) $T_{r}$ is firmly nonexpansive, i.e., for any $x, y \in H$

$$
\left\|T_{r} x-T_{r} y\right\|^{2} \leq\left\langle T_{r} x-T_{r} y, x-y\right\rangle ;
$$

3) $F\left(T_{r}\right)=E P(G)$;

4) $E P(G)$ is closed and convex.

Lemma 2.13 [18] Let $H, K, G$ and $T_{r} x$ be as in Lemma 2.12. Then we have

$$
\left\|T_{s} x-T_{t} x\right\|^{2} \leq \frac{s-t}{s}\left\langle T_{s} x-T_{t} x, T_{s} x-x\right\rangle,
$$

for all $s, t>0$ and $x \in H$.

\section{Main Results}

Theorem 3.1 Let $K$ be a nonempty closed convex subset of a Hilbert space $H$. Let $S$ be a semigroup, $\mathcal{S}=\{T(t): t \in S\}$ be a nonexpansive semigroup on $S$, $G: K \times K \rightarrow \mathbb{R}$ be a bifunction satisfying $(A 1)-(A 4)$ and $A: K \rightarrow H$ be an $\alpha-$ inverse strongly monotone mapping with

$$
\mathcal{F}:=F(\mathcal{S}) \cap G M E P(G, A, \varphi) \neq \varnothing .
$$

Let $\varphi: K \rightarrow \mathbb{R} \cup\{+\infty\}$ be a proper lower semicontinuous and convex function, $X$ be a left invariant subspace of $\ell^{\infty}(S)$ such that $1 \in X$ and the function $t \rightarrow\langle T(t) x, y\rangle$ be an element of $X$ for each $x, y \in K$. Let $\left\{\mu_{n}\right\}$ be a left regular sequence of means on $X$ such that $\left\|\mu_{n+1}-\mu_{n}\right\| \rightarrow 0$ as $n \rightarrow \infty$ and $f: K \rightarrow K$ be a Meir-Keeler contraction. Let $\left\{x_{n}\right\}$ be the sequence generated by $x_{1} \in K$ and

$$
\left\{\begin{array}{l}
u_{n} \in K \text { such that } \\
G\left(u_{n}, y\right)+\left\langle A x_{n}, y-u_{n}\right\rangle+\frac{1}{r_{n}}\left\langle y-u_{n}, u_{n}-x_{n}\right\rangle+\varphi(y)-\varphi\left(u_{n}\right) \geq 0, \forall y \in K, \\
x_{n+1}=\beta_{n} x_{n}+\beta_{n}^{\prime} T_{\mu_{n}}\left[\alpha_{n} f\left(x_{n}\right)+\left(1-\alpha_{n}\right) u_{n}\right]+\beta_{n}^{\prime \prime} e_{n}, n \geq 1,
\end{array}\right.
$$

where $\left\{e_{n}\right\}$ is bounded sequence in $K,\left\{\alpha_{n}\right\},\left\{\beta_{n}\right\},\left\{\beta_{n}^{\prime}\right\}$ and $\left\{\beta_{n}^{\prime \prime}\right\}$ are real number sequences in $(0,1)$ and $\left\{r_{n}\right\} \subset(0, \infty)$ satisfying the conditions.

(C1) $\beta_{n}+\beta_{n}^{\prime}+\beta_{n}^{\prime \prime}=1,0<a \leq \beta_{n} \leq b<1$;

(C2) $\lim _{n \rightarrow \infty} \alpha_{n}=0, \sum_{n=1}^{\infty} \alpha_{n}=\infty$;

(C3) $0<\liminf _{n \rightarrow \infty} \alpha_{n} \leq \limsup _{n \rightarrow \infty} \alpha_{n}<1$;

(C4) $0<\liminf _{n \rightarrow \infty} r_{n} \leq \limsup _{n \rightarrow \infty} r_{n}<2 \alpha, \sum_{n=1}^{\infty}\left|\beta_{n}^{\prime \prime}\right|<\infty, \lim _{n \rightarrow \infty}\left|r_{n+1}-r_{n}\right|=0$.

Then the sequence $\left\{x_{n}\right\}$ strongly converges to $p \in \mathcal{F}$ which is also solves the following variational inequality problem: 


$$
\langle f(p)-p, q-p\rangle \leq 0, \forall q \in \mathcal{F} .
$$

Proof. We give the several steps for the proof.

Step 1: First we show that $\left\{x_{n}\right\}$ is bounded. Put $u_{n}=T_{r_{n}}\left(x_{n}-r_{n} A x_{n}\right)$ and $y_{n}=\alpha_{n} f\left(x_{n}\right)+\left(1-\alpha_{n}\right) u_{n}$ for all $n \geq 1$. Then for $w \in \mathcal{F}$, we have

$$
\begin{aligned}
\left\|u_{n}-w\right\|^{2} & =\left\|T_{r_{n}}\left(x_{n}-r_{n} A x_{n}\right)-T_{r_{n}}\left(w-r_{n} A w\right)\right\|^{2} \\
& \leq\left\|\left(x_{n}-r_{n} A x_{n}\right)-\left(w-r_{n} A w\right)\right\|^{2} \\
& \leq\left\|\left(x_{n}-w\right)-r_{n}\left(A x_{n}-A w\right)\right\|^{2} \\
& \leq\left\|x_{n}-w\right\|^{2}-2 r_{n}\left\langle x_{n}-w, A x_{n}-A w\right\rangle+r_{n}^{2}\left\|A x_{n}-A w\right\|^{2} \\
& \leq\left\|x_{n}-w\right\|^{2}-2 r_{n} \alpha\left\|A x_{n}-A w\right\|^{2}+r_{n}^{2}\left\|A x_{n}-A w\right\|^{2} \\
& \leq\left\|x_{n}-w\right\|^{2}+r_{n}\left(r_{n}-2 \alpha\right)\left\|A x_{n}-A w\right\|^{2} \\
& \leq\left\|x_{n}-w\right\|^{2} .
\end{aligned}
$$

Set $T_{n}=\alpha_{n} I+\left(1-\alpha_{n}\right) T$, then $T_{n}$ is nonexpansive and $F\left(T_{n}\right)=F(T)$. Hence we have

$$
\begin{aligned}
& \left\|x_{n+1}-w\right\| \\
& \leq \beta_{n}\left\|x_{n}-w\right\|+\beta_{n}^{\prime}\left\|T_{\mu_{n}} y_{n}-w\right\|+\beta_{n}^{\prime \prime}\left\|e_{n}-w\right\| \\
& \leq \beta_{n}\left\|x_{n}-w\right\|+\beta_{n}^{\prime}\left\|y_{n}-w\right\|+\beta_{n}^{\prime \prime}\left\|e_{n}-w\right\| \\
& \leq \beta_{n}\left\|x_{n}-w\right\|+\beta_{n}^{\prime}\left(\alpha_{n}\left\|f\left(x_{n}\right)-w\right\|+\left(1-\alpha_{n}\right)\left\|u_{n}-w\right\|\right)+\beta_{n}^{\prime \prime}\left\|e_{n}-w\right\| \\
& \leq \beta_{n}\left\|x_{n}-w\right\|+\beta^{\prime}\left(\alpha_{n}\left\|f\left(x_{n}\right)-f(w)\right\|+\alpha_{n}\|f(w)-w\|+\left(1-\alpha_{n}\right)\left\|x_{n}-w\right\|\right) \\
& \quad+\beta_{n}^{\prime \prime}\left\|e_{n}-w\right\| \\
& \leq \beta_{n}\left\|x_{n}-w\right\|+\beta^{\prime}\left(\alpha_{n} \psi\left(\left\|x_{n}-w\right\|\right)+\alpha_{n}\|f(w)-w\|+\left(1-\alpha_{n}\right)\left\|x_{n}-w\right\|\right) \\
& \quad+\beta_{n}^{\prime \prime}\left\|e_{n}-w\right\| \\
& \leq\left\|x_{n}-w\right\|-\alpha_{n}\left(\beta^{\prime}\left(\eta\left(\left\|x_{n}-w\right\|\right)\right)+\alpha_{n} \beta^{\prime} \eta\left(\eta^{-1}(\|f(w)-w\|)\right)+\beta_{n}^{\prime \prime}\left\|e_{n}-w\right\|\right. \\
& \leq \max \left\{\left\|x_{n}-w\right\|, \eta^{-1}(\|f(w)-w\|),\left\|e_{n}-w\right\|\right\} .
\end{aligned}
$$

By induction, we can prove that

$$
\left\|x_{n}-w\right\| \leq \max \left\{\left\|x_{1}-w\right\|, \eta^{-1}(\|f(w)-w\|),\left\|e_{n}-w\right\|\right\}, \forall n \geq 1 .
$$

Hence the sequence $\left\{x_{n}\right\}$ is bounded. So $\left\{f\left(x_{n}\right)\right\},\left\{u_{n}\right\},\left\{y_{n}\right\}$ and $\left\{T_{\mu_{n}} y_{n}\right\}$ are all bounded.

Step 2: We next show that

$$
\lim _{n \rightarrow \infty}\left\|x_{n+1}-x_{n}\right\|=0
$$

Observe that

$$
\lim _{n \rightarrow \infty}\left\|T_{\mu_{n+1}} y_{n}-T_{\mu_{n}} y_{n}\right\|=0 .
$$

Indeed

$$
\begin{aligned}
& \left\|T_{\mu_{n+1}} y_{n}-T_{\mu_{n}} y_{n}\right\| \sup _{\|z\|=1}\left|\left\langle T_{\mu_{n+1}} y_{n}-T_{\mu_{n}} y_{n}, z\right\rangle\right| \\
& =\sup _{\|z\|=1}\left|\left(\mu_{n+1}\right)_{s}\left\langle T(s) y_{n}, z\right\rangle-\left(\mu_{n}\right)_{s}\left\langle T(s) y_{n}, z\right\rangle\right| \\
& \leq\left\|\mu_{n+1}-\mu_{n}\right\| \sup _{s \in S}\left\|T(s) y_{n}\right\| .
\end{aligned}
$$


Since $\left\{y_{n}\right\}$ is bounded and $\lim _{n \rightarrow \infty}\left\|\mu_{n+1}-\mu_{n}\right\|=0, \quad$ (3.3) holds. Since $u_{n}=T_{r_{n}}\left(x_{n}-r_{n} A x_{n}\right)$ and $u_{n+1}=T_{r_{n+1}}\left(x_{n+1}-r_{n+1} A x_{n+1}\right)$, we have

$$
\begin{aligned}
& \left\|u_{n+1}-u_{n}\right\|=\left\|T_{r_{n+1}}\left(x_{n+1}-r_{n+1} A x_{n+1}\right)-T_{r_{n}}\left(x_{n}-r_{n} A x_{n}\right)\right\| \\
& \leq\left\|T_{r_{n+1}}\left(x_{n+1}-r_{n+1} A x_{n+1}\right)-T_{r_{n+1}}\left(x_{n}-r_{n} A x_{n}\right)\right\| \\
& \quad+\left\|T_{r_{n+1}}\left(x_{n}-r_{n} A x_{n}\right)-T_{r_{n}}\left(x_{n}-r_{n} A x_{n}\right)\right\| \\
& \leq\left\|\left(x_{n+1}-r_{n+1} A x_{n+1}\right)-\left(x_{n}-r_{n} A x_{n}\right)\right\|+\left\|T_{r_{n+1}}\left(x_{n}-r_{n} A x_{n}\right)-T_{r_{n}}\left(x_{n}-r_{n} A x_{n}\right)\right\| \\
& \leq\left\|\left(x_{n+1}-r_{n+1} A x_{n+1}\right)-\left(x_{n}-r_{n+1} A x_{n}\right)\right\|+\left\|\left(x_{n}-r_{n+1} A x_{n}\right)-\left(x_{n}-r_{n} A x_{n}\right)\right\| \\
& \quad+\left\|T_{r_{n+1}}\left(x_{n}-r_{n} A x_{n}\right)-T_{r_{n}}\left(x_{n}-r_{n} A x_{n}\right)\right\| \\
& \leq\left\|x_{n+1}-x_{n}\right\|+\mid r_{n+1}-r_{n}\left\|A x_{n}\right\|+\left\|T_{r_{n+1}}\left(x_{n}-r_{n} A x_{n}\right)-T_{r_{n}}\left(x_{n}-r_{n} A x_{n}\right)\right\| .
\end{aligned}
$$

From $y_{n}=\alpha_{n} f\left(x_{n}\right)+\left(1-\alpha_{n}\right) u_{n}$ and $y_{n+1}=\alpha_{n+1} f\left(x_{n+1}\right)+\left(1-\alpha_{n+1}\right) u_{n+1}$, we have

$$
\begin{aligned}
y_{n+1}-y_{n} & =\alpha_{n+1} f\left(x_{n+1}\right)+\left(1-\alpha_{n+1}\right) u_{n+1}-\left(\alpha_{n} f\left(x_{n}\right)+\left(1-\alpha_{n}\right) u_{n}\right) \\
& =\alpha_{n+1}\left(f\left(x_{n+1}\right)-u_{n+1}\right)+\alpha_{n}\left(u_{n}-f\left(x_{n}\right)\right)+\left(u_{n+1}-u_{n}\right),
\end{aligned}
$$

it follows that

$$
\left\|y_{n+1}-y_{n}\right\| \leq \alpha_{n+1}\left(\left\|f\left(x_{n+1}\right)\right\|+\left\|u_{n+1}\right\|\right)+\alpha_{n}\left(\left\|u_{n}\right\|+\left\|f\left(x_{n}\right)\right\|\right)+\left\|u_{n+1}-u_{n}\right\| .
$$

We see that

$$
\begin{aligned}
\left\|T_{\mu_{n+1}} y_{n+1}-T_{\mu_{n}} y_{n}\right\| & \leq\left\|T_{\mu_{n+1}} y_{n+1}-T_{\mu_{n+1}} y_{n}\right\|+\left\|T_{\mu_{n+1}} y_{n}-T_{\mu_{n}} y_{n}\right\| \\
& \leq\left\|y_{n+1}-y_{n}\right\|+\left\|T_{\mu_{n+1}} y_{n}-T_{\mu_{n}} y_{n}\right\| .
\end{aligned}
$$

Combining (3.4) and (3.5) with (3.6), we obtain

$$
\begin{aligned}
\left\|T_{\mu_{n+1}} y_{n+1}-T_{\mu_{n}} y_{n}\right\| \leq & \alpha_{n+1}\left(\left\|f\left(x_{n+1}\right)\right\|+\left\|u_{n+1}\right\|\right)+\alpha_{n}\left(\left\|u_{n}\right\|+\left\|f\left(x_{n}\right)\right\|\right) \\
& +\left\|x_{n+1}-x_{n}\right\|+\mid r_{n+1}-r_{n}\left\|A x_{n}\right\| \\
& +\left\|T_{r_{n+1}}\left(x_{n}-r_{n} A x_{n}\right)-T_{r_{n}}\left(x_{n}-r_{n} A x_{n}\right)\right\|+\left\|T_{\mu_{n+1}} y_{n}-T_{\mu_{n}} y_{n}\right\| .
\end{aligned}
$$

Using Lemma 2.13, (3.3), (C1) and (C4), then we have

$$
\limsup _{n \rightarrow \infty}\left(\left\|T_{\mu_{n+1}} y_{n+1}-T_{\mu_{n}} y_{n}\right\|-\left\|x_{n+1}-x_{n}\right\|\right) \leq 0 \text {. }
$$

From this inequality and ( $C 3)$, it follows from Lemma 2.9 that

$$
\lim _{n \rightarrow \infty}\left\|T_{\mu_{n}} y_{n}-x_{n}\right\|=0 \text {. }
$$

It implies that

$$
\lim _{n \rightarrow \infty}\left\|x_{n+1}-x_{n}\right\|=0 .
$$

Step 3: Next we prove that for all $t \in S$,

$$
\lim _{n \rightarrow \infty}\left\|x_{n+1}-T(t) x_{n}\right\|=0 \text {. }
$$

Put

$$
M=\max \left\{\|x-w\|, \eta^{-1}(\|f(w)-w\|),\left\|e_{n}-w\right\|\right\} .
$$

Set $D=\{y \in K:\|y-w\| \leq M\}$. It is easily seen that $D$ is a nonempty bounded closed convex subset of $K$. Further $\left\{x_{n}\right\},\left\{y_{n}\right\}$ and $\left\{u_{n}\right\}$ are in $D$. To complete 
our proof, we follows that proof line as in [30]. From [40], for every $\epsilon>0$ there exists $\delta>0$ such that for all $t \in S$,

$$
\overline{c o} F_{\delta}(T(t) ; D)+B_{\delta} \subseteq F_{\epsilon}(T(t) ; D) .
$$

From Corollary 1.1 in [40], there exists a natural number $N$ such that for all $t, s \in S, y \in D$,

$$
\left\|\frac{1}{N+1} \sum_{i=0}^{N} T\left(t^{i} s\right) y-T(t)\left(\frac{1}{N+1} \sum_{i=0}^{N} T\left(t^{i} s\right) y\right)\right\| \leq \delta .
$$

Since $\left\{\mu_{n}\right\}$ is left regular, for $t \in S$ there exists $n_{0} \in N$ such that

$$
\left\|\mu_{n}-\ell_{t}^{*} \mu_{n}\right\| \leq \frac{\delta}{2(M+\|w\|)}
$$

for all $n \geq n_{0}$ and $i=1,2, \cdots, N$. Therefore, we have for all $n \geq n_{0}$,

$$
\begin{aligned}
& \sup _{y \in D}\left\|T_{\mu_{n}} y-\int \frac{1}{N+1} \sum_{i=0}^{N} T\left(t^{i} s\right) y d \mu_{n}(s)\right\| \\
& =\operatorname{supsup}_{y \in D\|z\|=1}\left|\left(\mu_{n}\right)_{s}\langle T(s) y, z\rangle-\left(\mu_{n}\right)_{s}\left\langle\frac{1}{N+1} \sum_{i=0}^{N} T\left(t^{i} s\right) y, z\right\rangle\right| \\
& \leq \frac{1}{N+1} \sum_{i=0}^{N} \operatorname{supsup}_{y \in D\|z\|=1}\left|\left(\mu_{n}\right)_{s}\langle T(s) y, z\rangle-\left(\ell_{t^{*}}^{*} \mu_{n}\right)_{s}\langle T(s) y, z\rangle\right| \\
& \leq \max _{i=1,2, \cdots, N}\left\|\mu_{n}-\ell_{i^{*}}^{*} \mu_{n}\right\|(M+\|w\|) \leq \frac{\delta}{3} .
\end{aligned}
$$

We observe from Lemma 2.2 (iii) that

$$
\int \frac{1}{N+1} \sum_{i=0}^{N} T\left(t^{i} s\right) y d \mu_{n}(s) \in \overline{c o}\left\{\frac{1}{N+1} \sum_{i=0}^{N} T\left(t^{i}\right) T(s) y: s \in S\right\} .
$$

Combining (3.10), (3.12) and (3.12), we have for all $y \in D, n \geq n_{0}$,

$$
\begin{aligned}
T_{\mu_{n}} y & =\int \frac{1}{N+1} \sum_{i=0}^{N} T\left(t^{i} s\right) y d \mu_{n}(s)+\left(T_{\mu_{n}} y-\int \frac{1}{N+1} \sum_{i=0}^{N} T\left(t^{i} s\right) y d \mu_{n}(s)\right) \\
& \in \overline{c o}\left\{\frac{1}{N+1} \sum_{i=0}^{N} T\left(t^{i}\right) T(s) y: s \in S\right\}+B_{\frac{\delta}{3}} \\
& \subseteq \overline{c o} F_{\delta}(T(t) ; D)+B_{\frac{\delta}{3}} .
\end{aligned}
$$

Let $t \in S$ and $\epsilon>0$. Then there exists $\delta>0$ which satisfies (3.9). From (C3) there exist $a, b \in(0,1)$ such that $0<a \leq \beta_{n} \leq b<1$. From (3.7) there exists $k_{0} \in N$ such that $\left\|x_{n}-T_{\mu_{n}} y_{n}\right\|<\frac{\delta}{3 b}$ and $\left\|e_{n}-T_{\mu_{n}} y_{n}\right\|<\frac{\delta}{3 b}$, for all $n>k_{0}$. So from (3.9) and (3.13), we have

$$
\begin{aligned}
x_{n+1} & =\beta_{n} x_{n}+\beta_{n}^{\prime} T_{\mu_{n}} y_{n}+\beta_{n}^{\prime \prime} e_{n} \\
& =\beta_{n} x_{n}+\left(1-\beta_{n}\right) T_{\mu_{n}} y_{n}+\beta_{n}^{\prime \prime}\left(e_{n}-T_{\mu_{n}} y_{n}\right) \\
& =T_{\mu_{n}} y_{n}+\beta_{n}\left(x_{n}-T_{\mu_{n}} y_{n}\right)+\beta_{n}^{\prime \prime}\left(e_{n}-T_{\mu_{n}} y_{n}\right) \\
& \in \overline{c o} F_{\delta}(T(t) ; D)+B_{\frac{\delta}{3}}+B_{\frac{\delta}{3}}+B_{\frac{\delta}{3}} \\
& \subseteq \overline{c o} F_{\delta}(T(t) ; D)+B_{\delta} \subseteq F_{\varepsilon}(T(t) ; D) .
\end{aligned}
$$


Hence $\limsup _{n \rightarrow \infty}\left\|x_{n}-T(t) x_{n}\right\| \leq \epsilon$. Since $\epsilon>0$ is arbitrary,

$$
\lim _{n \rightarrow \infty}\left\|x_{n}-T(t) x_{n}\right\|=0 .
$$

Step 4: We next show that

$$
\lim _{n \rightarrow \infty}\left\|u_{n}-x_{n}\right\|=0
$$

Using inequality (3.2), we obtain

$$
\begin{aligned}
& \left\|x_{n+1}-w\right\|^{2} \leq \beta_{n}\left\|x_{n}-w\right\|^{2}+\beta_{n}^{\prime}\left\|T_{\mu_{n}} y_{n}-w\right\|^{2}+\beta_{n}^{\prime \prime}\left\|e_{n}-w\right\|^{2} \\
& \leq \beta_{n}\left\|x_{n}-w\right\|^{2}+\beta_{n}^{\prime}\left\|y_{n}-w\right\|^{2}+\beta_{n}^{\prime \prime}\left\|e_{n}-w\right\|^{2} \\
& \leq \beta_{n}\left\|x_{n}-w\right\|^{2}+\beta_{n}^{\prime}\left(\alpha_{n}\left\|f\left(x_{n}\right)-w\right\|^{2}+\left(1-\alpha_{n}\right)\left\|u_{n}-w\right\|^{2}\right)+\beta_{n}^{\prime \prime}\left\|e_{n}-w\right\|^{2} \\
& \leq \beta_{n}\left\|x_{n}-w\right\|^{2}+\beta_{n}^{\prime}\left(\alpha_{n}\left\|f\left(x_{n}\right)-w\right\|^{2}\right. \\
& \left.\quad+\left(1-\alpha_{n}\right)\left(\left\|x_{n}-w\right\|^{2}+r_{n}\left(r_{n}-2 \alpha\right)\left\|A x_{n}-A w\right\|^{2}\right)\right)+\beta_{n}^{\prime \prime}\left\|e_{n}-w\right\|^{2} \\
& \leq\left\|x_{n}-w\right\|^{2}+\beta_{n}^{\prime} \alpha_{n}\left\|f\left(x_{n}\right)-w\right\|^{2} \\
& \quad+\beta_{n}^{\prime}\left(1-\alpha_{n}\right) r_{n}\left(r_{n}-2 \alpha\right)\left\|A x_{n}-A w\right\|^{2}+\beta_{n}^{\prime \prime}\left\|e_{n}-w\right\|^{2},
\end{aligned}
$$

which implies that

$$
\begin{aligned}
& \beta_{n}^{\prime}\left(1-\alpha_{n}\right) r_{n}\left(2 \alpha-r_{n}\right)\left\|A x_{n}-A w\right\|^{2} \\
& \leq\left\|x_{n}-w\right\|^{2}-\left\|x_{n+1}-w\right\|^{2}+\beta_{n}^{\prime} \alpha_{n}\left\|f\left(x_{n}\right)-w\right\|^{2}+\beta_{n}^{\prime \prime}\left\|e_{n}-w\right\|^{2} .
\end{aligned}
$$

From (C1)-(C4) and (3.8), we obtain

$$
\lim _{N \rightarrow \infty}\left\|A x_{n}-A w\right\|=0
$$

Since $T_{r_{n}}$ is firmly nonexpansive,

$$
\begin{aligned}
\left\|u_{n}-w\right\|^{2}= & \left\|T_{r_{n}}\left(x_{n}-r_{n} A x_{n}\right)-T_{r_{n}}\left(w-r_{n} A w\right)\right\|^{2} \\
\leq & \left\langle x_{n}-r_{n} A x_{n}-\left(w-r_{n} A w\right), u_{n}-w\right\rangle \\
= & \frac{1}{2}\left(\left\|\left(x_{n}-r_{n} A x_{n}\right)-\left(w-r_{n} A w\right)\right\|^{2}+\left\|u_{n}-w\right\|^{2}\right. \\
& \left.-\left\|\left(x_{n}-r_{n} A x_{n}\right)-\left(w-r_{n} A w\right)-\left(u_{n}-w\right)\right\|^{2}\right) \\
\leq & \frac{1}{2}\left(\left\|x_{n}-w\right\|^{2}+\left\|u_{n}-w\right\|^{2}-\left\|\left(x_{n}-u_{n}\right)-r_{n}\left(A x_{n}-A w\right)\right\|^{2}\right) \\
\leq & \frac{1}{2}\left(\left\|x_{n}-w\right\|^{2}+\left\|u_{n}-w\right\|^{2}-\left\|x_{n}-u_{n}\right\|^{2}\right. \\
& \left.+2 r_{n}\left\langle x_{n}-u_{n}, A x_{n}-A w\right\rangle-r_{n}^{2}\left\|A x_{n}-A w\right\|^{2}\right) .
\end{aligned}
$$

Therefore

$$
\begin{aligned}
\left\|u_{n}-w\right\|^{2} \leq & \left\|x_{n}-w\right\|^{2}-\left\|x_{n}-u_{n}\right\|^{2} \\
& +2 r_{n}\left\langle x_{n}-u_{n}, A x_{n}-A w\right\rangle-r_{n}^{2}\left\|A x_{n}-A w\right\|^{2} .
\end{aligned}
$$

Then we have 


$$
\begin{aligned}
& \left\|x_{n+1}-w\right\|^{2} \leq \beta_{n}\left\|x_{n}-w\right\|^{2}+\beta_{n}^{\prime}\left\|T_{\mu_{n}} y_{n}-w\right\|^{2}+\beta_{n}^{\prime \prime}\left\|e_{n}-w\right\|^{2} \\
& \leq \beta_{n}\left\|x_{n}-w\right\|^{2}+\beta_{n}^{\prime}\left\|y_{n}-w\right\|^{2}+\beta_{n}^{\prime \prime}\left\|e_{n}-w\right\|^{2} \\
& \leq \beta_{n}\left\|x_{n}-w\right\|^{2}+\beta_{n}^{\prime}\left(\alpha_{n}\left\|f\left(x_{n}\right)-w\right\|^{2}+\left(1-\alpha_{n}\right)\left\|u_{n}-w\right\|^{2}\right)+\beta_{n}^{\prime \prime}\left\|e_{n}-w\right\|^{2} \\
& \leq \beta_{n}\left\|x_{n}-w\right\|^{2}+\beta_{n}^{\prime}\left(\alpha_{n}\left\|f\left(x_{n}\right)-w\right\|^{2}\right. \\
& \left.\quad+\left(1-\alpha_{n}\right)\left(\left\|x_{n}-w\right\|^{2}-\left\|x_{n}-u_{n}\right\|^{2}+2 r_{n}\left\langle x_{n}-u_{n}, A x_{n}-A w\right\rangle-r_{n}^{2}\left\|A x_{n}-A w\right\|^{2}\right)\right) \\
& \quad+\beta_{n}^{\prime \prime}\left\|e_{n}-w\right\|^{2} \\
& \leq\left\|x_{n}-w\right\|^{2}+\alpha_{n}\left\|f\left(x_{n}\right)-w\right\|^{2}-\beta_{n}^{\prime}\left\|x_{n}-u_{n}\right\|^{2} \\
& \quad+2\left(1-\alpha_{n}\right) r_{n}\left\|x_{n}-u_{n}\right\|\left\|A x_{n}-A w\right\|+\beta_{n}^{\prime \prime}\left\|e_{n}-w\right\|^{2},
\end{aligned}
$$

which yields

$$
\begin{aligned}
\beta_{n}^{\prime}\left\|x_{n}-u_{n}\right\|^{2} \leq & \left\|x_{n}-w\right\|^{2}-\left\|x_{n+1}-w\right\|^{2}+\alpha_{n}\left\|f\left(x_{n}\right)-w\right\|^{2} \\
& +2 \beta_{n}^{\prime} r_{n}\left\|x_{n}-u_{n}\right\|\left\|A x_{n}-A w\right\|+\beta_{n}^{\prime \prime}\left\|e_{n}-w\right\|^{2} .
\end{aligned}
$$

Hence, from (C2), (C3) and (3.16) we obtain

$$
\lim _{n \rightarrow \infty}\left\|u_{n}-x_{n}\right\|=0 .
$$

Since $y_{n}=\alpha_{n} f\left(x_{n}\right)+\left(1-\alpha_{n}\right) u_{n}$, we have $y_{n}-u_{n}=\alpha_{n}\left(f\left(x_{n}\right)-u_{n}\right)$ and hence

$$
\lim _{n \rightarrow \infty}\left\|y_{n}-u_{n}\right\|=0 \text {. }
$$

On the other hand, by Proposition 2.7 (i), we know that $P_{\mathcal{F}} f$ is a Meir-Keeler contraction. From Theorem 2.4, there exists a unique element $p$ such that $P_{\mathcal{F}} f(p)=p$ which is equivalent to

$$
\langle f(p)-p, q-p\rangle \leq 0, \forall q \in \mathcal{F} .
$$

Step 5: We next show that

$$
\limsup _{n \rightarrow \infty}\left\langle f(p)-p, y_{n}-p\right\rangle \leq 0 .
$$

To see this, we chose a subsequence $\left\{y_{n_{k}}\right\}$ of $\left\{y_{n}\right\}$ such that

$$
\limsup _{n \rightarrow \infty}\left\langle f(p)-p, y_{n}-p\right\rangle=\lim _{k \rightarrow \infty}\left\langle f(p)-p, y_{n_{k}}-p\right\rangle \text {. }
$$

Since $\left\{x_{n}\right\}$ is a bounded, $K$ is closed and $H$ is reflexive, there exists a point $z \in K$ such that $x_{n_{k}} \rightarrow z \in K$. From (3.17) and (3.18) there exists a corresponding subsequence $\left\{u_{n_{k}}\right\}$ of $\left\{u_{n}\right\}$ (resp. $\left\{y_{n_{k}}\right\}$ of $\left\{y_{n}\right\}$ ) such that $u_{n_{k}} \rightarrow z \in K \quad$ (resp. $\left.\quad y_{n_{k}} \rightarrow z \in K\right)$. We next show that $z \in \operatorname{GMEP}(G, A, \varphi)$. Since $u_{n}=T_{r_{n}}\left(x_{n}-r_{n} A x_{n}\right)$. We can write

$$
G\left(u_{n}, y\right)+\left\langle A x_{n}, y-u_{n}\right\rangle+\frac{1}{r_{n}}\left\langle y-u_{n}, u_{n}-x_{n}\right\rangle+\varphi(y)-\varphi\left(u_{n}\right) \geq 0, \forall y \in K .
$$

From ( $A 2)$, we have

$$
\left\langle A x_{n}, y-u_{n}\right\rangle+\frac{1}{r_{n}}\left\langle y-u_{n}, u_{n}-x_{n}\right\rangle \geq G\left(y, u_{n}\right)-\varphi(y)+\varphi\left(u_{n}\right) \geq 0, \forall y \in K .
$$

Then 


$$
\begin{aligned}
& \left\langle A x_{n_{k}}, y-u_{n_{k}}\right\rangle+\frac{1}{r_{n_{k}}}\left\langle y-u_{n_{k}}, u_{n_{k}}-x_{n_{k}}\right\rangle \\
& \geq G\left(y, u_{n_{k}}\right)-\varphi(y)+\varphi\left(u_{n_{k}}\right) \geq 0, \forall y \in K .
\end{aligned}
$$

Put $y_{t}=t y+(1-t) z$, for $t \in(0,1]$ and $y \in K$. Since $y \in K$ and $z \in K$, $y_{t} \in K$. So from (3.19) we have

$$
\begin{aligned}
\left\langle y_{t}-u_{n_{k}}, A y_{t}\right\rangle \geq & \left\langle y_{t}-u_{n_{k}}, A y_{t}\right\rangle-\left\langle y_{t}-u_{n_{k}}, A x_{n_{k}}\right\rangle-\left\langle y_{t}-u_{n_{k}}, \frac{u_{n_{k}}-x_{n_{k}}}{r_{n_{k}}}\right\rangle \\
& +G\left(y_{t}, u_{n_{k}}\right)+\varphi\left(y_{t}\right)-\varphi\left(u_{n_{k}}\right) \\
= & \left\langle y_{t}-u_{n_{k}}, A y_{t}-A u_{n_{k}}\right\rangle+\left\langle y_{t}-u_{n_{k}}, A u_{n_{k}}-A x_{n_{k}}\right\rangle \\
& -\left\langle y_{t}-u_{n_{k}}, \frac{u_{n_{k}}-x_{n_{k}}}{r_{n_{k}}}\right\rangle+G\left(y_{t}, u_{n_{k}}\right)+\varphi\left(y_{t}\right)-\varphi\left(u_{n_{k}}\right) \\
\geq & \left\langle y_{t}-u_{n_{k}}, A u_{n_{k}}-A x_{n_{k}}\right\rangle-\left\langle y_{t}-u_{n_{k}}, \frac{u_{n_{k}}-x_{n_{k}}}{r_{n_{k}}}\right\rangle \\
& +G\left(y_{t}, u_{n_{k}}\right)+\varphi\left(y_{t}\right)-\varphi\left(u_{n_{k}}\right)
\end{aligned}
$$

From (A4), we have

$$
\left\langle y_{t}-z, A y_{t}\right\rangle \geq G\left(y_{t}, z\right)+\varphi\left(y_{t}\right)-\varphi(z) .
$$

From (A1)-(A4) and (3.20), we have

$$
\begin{aligned}
0 & =G\left(y_{t}, y_{t}\right) \\
& \leq t G\left(y_{t}, y\right)+(1-t) G\left(y_{t}, z\right) \square \\
& \leq t G\left(y_{t}, y\right)+(1-t)\left\langle y_{t}-z, A y_{t}\right\rangle \\
& \leq t G\left(y_{t}, y\right)+(1-t)\left\langle y-z, A y_{t}\right\rangle .
\end{aligned}
$$

It follows that

$$
0 \leq t G\left(y_{t}, y\right)+(1-t)\left\langle y-z, A y_{t}\right\rangle,
$$

letting $t \rightarrow 0$ by $(A 3)$, we have

$$
0 \leq G(z, y)+\langle y-z, A z\rangle, \forall y \in K .
$$

Hence $z \in G M E P(G, A, \varphi)$. It is easily seen that $z \in F(\mathcal{S})$. Indeed, since $x_{n_{k}} \rightarrow z$ and $\left\|x_{n}-T(t) x_{n}\right\| \rightarrow 0$, for all $t \in S$, we conclude from Lemma 2.1 that $z \in F(\mathcal{S})$. Consequently, we have $z \in \mathcal{F}=F(\mathcal{S}) \cap \operatorname{GMEP}(G, A, \varphi)$ and hence

$$
\begin{aligned}
\limsup _{n \rightarrow \infty}\left\langle f(p)-p, y_{n}-p\right\rangle & =\lim _{k \rightarrow \infty}\left\langle f(p)-p, y_{n_{k}}-p\right\rangle \\
& =\langle f(p)-p, z-p\rangle \\
& \leq 0 .
\end{aligned}
$$

Step 6: Now we are in a position to show that $x$ is a fixed point of $T$.

Let $\lim _{n \rightarrow \infty}\left\|x_{n}-w\right\|=d>0$. Then we have

$$
\begin{aligned}
& \lim _{n \rightarrow \infty}\left\|x_{n+1}-w\right\| \\
& =\lim _{n \rightarrow \infty}\left\|\beta_{n}\left(x_{n}-w+\beta_{n}^{\prime \prime}\left(e_{n}-T_{\mu_{n}} u_{n}\right)\right)+\left(1-\beta_{n}\right)\left(T_{\mu_{n}} u_{n}-w+\beta_{n}^{\prime \prime}\left(e_{n}-T_{\mu_{n}} u_{n}\right)\right)\right\| \\
& =d .
\end{aligned}
$$


We note that

$$
\begin{aligned}
& \limsup _{n \rightarrow \infty}\left\|x_{n}-w+\beta_{n}^{\prime \prime}\left(e_{n}-T_{\mu_{n}} u_{n}\right)\right\| \\
& \leq \underset{n \rightarrow \infty}{\limsup }\left\|x_{n}-w\right\|+\limsup _{n \rightarrow \infty} \beta^{\prime \prime}\left\|e_{n}-T_{\mu_{n}} u_{n}\right\| \\
& \leq d
\end{aligned}
$$

and

$$
\begin{aligned}
& \limsup _{n \rightarrow \infty}\left\|T_{\mu_{n}} u_{n}-w+\beta_{n}^{\prime \prime}\left(e_{n}-T_{\mu_{n}} u_{n}\right)\right\| \\
& \leq \underset{n \rightarrow \infty}{\limsup }\left\|T_{\mu_{n}} u_{n}-w\right\|+\underset{n \rightarrow \infty}{\limsup } \beta_{n}^{\prime \prime}\left\|e_{n}-T_{\mu_{n}} u_{n}\right\| \\
& \leq \underset{n \rightarrow \infty}{\limsup }\left\|u_{n}-w\right\|+\underset{n \rightarrow \infty}{\limsup } \beta_{n}^{\prime \prime}\left\|e_{n}-u_{n}\right\| \leq d .
\end{aligned}
$$

It follows from Lemma 2.10 that

$$
\lim _{n \rightarrow \infty}\left\|x_{n}-T_{\mu_{n}} u_{n}\right\|=0 .
$$

On the other hand, we have

$$
\begin{aligned}
\left\|T_{\mu_{n}} x_{n}-x_{n}\right\| & \leq\left\|T_{\mu_{n}} x_{n}-T_{\mu_{n}} u_{n}\right\|+\left\|T_{\mu_{n}} u_{n}-x_{n}\right\| \\
& \leq\left\|x_{n}-u_{n}\right\|+\left\|T_{\mu_{n}} u_{n}-x_{n}\right\| .
\end{aligned}
$$

It follows from (3.17) and (3.22) that

$$
\lim _{n \rightarrow \infty}\left\|x_{n}-T_{\mu_{n}} x_{n}\right\|=0 .
$$

Therefore $x \in F(T)$. Let $\left\{x_{n_{j}}\right\}$ be an another subsequence of $\left\{x_{n}\right\}$ converging to $x_{0}$ with $x_{0} \neq x$. Similarly, we can find $x_{0} \in F(T)$. Hence we have

$$
\begin{aligned}
d & =\liminf _{i \rightarrow \infty}\left\|x_{n_{i}}-x\right\|<\liminf _{i \rightarrow \infty}\left\|x_{n_{i}}-x_{0}\right\|=\liminf _{j \rightarrow \infty}\left\|x_{j}-x_{0}\right\| \\
& <\liminf _{j \rightarrow \infty}\left\|x_{j}-x\right\|=d .
\end{aligned}
$$

This is a contradiction. Hence we have $x=x_{0}$.

Step 7: We finally show that $x_{n} \rightarrow p$ as $n \rightarrow \infty$.

Suppose that $\left\{x_{n}\right\}$ does not strongly converge to $p \in \mathcal{F}$. Then there exists $\epsilon>0$ and a subsequence $\left\{x_{n_{j}}\right\}$ of $\left\{x_{n}\right\}$ such that $\left\|x_{n_{j}}-p\right\|>\epsilon$ for all $j \in\{0,-1, \cdots\}$. By Proposition 2.7, for this $\epsilon$ there exists $r \in(0,1)$ such that

$$
\left\|f\left(x_{n_{j}}\right)-f(p)\right\| \leq r\left\|x_{n_{j}}-p\right\| .
$$

So we have

$$
\begin{aligned}
& \left\|y_{n_{j}}-p\right\|^{2}=\left\|\alpha_{n_{j}}\left(f\left(x_{n_{j}}\right)-p\right)+\left(1-\alpha_{n_{j}}\right)\left(u_{n_{j}}-p\right)\right\|^{2} \\
& \leq\left(1-\alpha_{n_{j}}\right)^{2}\left\|u_{n_{j}}-p\right\|^{2}+2 \alpha_{n_{j}}\left\langle f\left(x_{n_{j}}\right)-p, y_{n_{j}}-p\right\rangle \\
& \leq\left(1-\alpha_{n_{j}}\right)^{2}\left\|x_{n_{j}}-p\right\|^{2}+2 \alpha_{n_{j}}\left\langle f\left(x_{n_{j}}\right)-f(p), y_{n_{j}}-p\right\rangle+2 \alpha_{n_{j}}\left\langle f(p)-p, y_{n_{j}}-p\right\rangle \\
& \leq\left(1-\alpha_{n_{j}}\right)^{2}\left\|x_{n_{j}}-p\right\|^{2}+2 \alpha_{n_{j}} r\left\|x_{n_{j}}-p\right\|\left\|y_{n_{j}}-p\right\|+2 \alpha_{n_{j}}\left\langle f(p)-p, y_{n_{j}}-p\right\rangle \\
& \leq\left(1-\alpha_{n_{j}}\right)^{2}\left\|x_{n_{j}}-p\right\|^{2}+2 \alpha_{n_{j}} r\left(\left\|x_{n_{j}}-p\right\|^{2}+\left\|y_{n_{j}}-p\right\|^{2}\right)+2 \alpha_{n_{j}}\left\langle f(p)-p, y_{n_{j}}-p\right\rangle .
\end{aligned}
$$


This implies that

$$
\left\|y_{n_{j}}-p\right\|^{2} \leq \frac{\left(1-\alpha_{n_{j}}\right)^{2}+\alpha_{n_{j}} r}{1-\alpha_{n_{j}} r}\left\|x_{n_{j}}-p\right\|^{2}+\frac{2 \alpha_{n_{j}}}{1-\alpha_{n_{j}} r}\left\langle f(p)-p, y_{n_{j}}-p\right\rangle .
$$

Hence

$$
\begin{aligned}
& \left\|x_{n_{j}+1}-p\right\|^{2} \leq \beta_{n_{j}}\left\|x_{n_{j}}-p\right\|^{2}+\beta_{n_{j}}^{\prime}\left\|T_{\mu_{n_{j}}} y_{n_{j}}-p\right\|^{2}+\beta_{n_{j}}^{\prime \prime}\left\|e_{n_{j}}-p\right\|^{2} \\
& \leq \beta_{n_{j}}\left\|x_{n_{j}}-p\right\|^{2}+\beta_{n_{j}}^{\prime}\left\|y_{n_{j}}-p\right\|^{2}+\beta_{n_{j}}^{\prime \prime}\left\|e_{n_{j}}-p\right\|^{2} \\
& \leq \beta_{n_{j}}\left\|x_{n_{j}}-p\right\|^{2}+\beta_{n_{j}}^{\prime}\left\{\frac{\left(1-\alpha_{n_{j}}\right)^{2}+\alpha_{n_{j}} r}{1-\alpha_{n_{j}} r}\left\|x_{n_{j}}-p\right\|^{2}+\frac{2 \alpha_{n_{j}}}{1-\alpha_{n_{j}} r}\left\langle f(p)-p, y_{n_{j}}-p\right\rangle\right\}+\beta_{n_{j}}^{\prime \prime}\left\|e_{n_{j}}-p\right\|^{2} \\
& \leq \beta_{n_{j}}\left\|x_{n_{j}}-p\right\|^{2}+\beta_{n_{j}}^{\prime}\left\{\frac{1-\alpha_{n_{j}} r-2(1-r) \alpha_{n_{j}}+\alpha_{n_{j}}^{2}}{1-\alpha_{n_{j}} r}\right\}\left\|x_{n_{j}}-p\right\|^{2}+\frac{2 \alpha_{n_{j}} \beta_{n_{j}}^{\prime}}{1-\alpha_{n_{j}} r}\left\langle f(p)-p, y_{n_{j}}-p\right\rangle+\beta_{n_{j}}^{\prime \prime}\left\|e_{n_{j}}-p\right\|^{2} \\
& =\beta_{n_{j}}\left\|x_{n_{j}}-p\right\|^{2}+\beta_{n_{j}}^{\prime}\left\{\left(1-\frac{2(1-r) \alpha_{n_{j}}}{1-\alpha_{n_{j}} r}\right)+\frac{\alpha_{n_{j}}^{2}}{1-\alpha_{n_{j}} r}\right\}\left\|x_{n_{j}}-p\right\|^{2}+\frac{2 \alpha_{n_{j}} \beta_{n_{j}}^{\prime}}{1-\alpha_{n_{j}} r}\left\langle f(p)-p, y_{n_{j}}-p\right\rangle+\beta_{n_{j}}^{\prime \prime}\left\|e_{n_{j}}-p\right\|^{2} \\
& \leq\left(1-\frac{2(1-r) \alpha_{n_{j}} \beta_{n_{j}}^{\prime}}{1-\alpha_{n_{j} r}}\right)\left\|x_{n_{j}}-p\right\|^{2}+\frac{2(1-r) \alpha_{n_{j}} \beta_{n_{j}}^{\prime}}{1-\alpha_{n_{j}} r}\left\{\frac{\alpha_{n_{j}}}{2(1-r)}\left\|x_{n_{j}}-p\right\|^{2}+\frac{1}{1-r}\left\langle f(p)-p, y_{n_{j}}-p\right\rangle\right\} \\
& +\beta_{n_{j}}^{\prime \prime}\left(\left\|e_{n_{j}}-p\right\|^{2}-\left\|x_{n_{j}}-p\right\|^{2}\right) \\
& =\left(1-\frac{2(1-r) \alpha_{n_{j}} \beta_{n_{j}}^{\prime}}{1-\alpha_{n_{j}} r}\right)\left\|x_{n_{j}}-p\right\|^{2}+\frac{2(1-r) \alpha_{n_{j}} \beta_{n_{j}}^{\prime}}{1-\alpha_{n_{j}} r}\left\{\left(\frac{\alpha_{n_{j}}}{2(1-r)}+\frac{\beta_{n_{j}}^{\prime \prime}\left(1-\alpha_{n_{j}} r\right)}{2(1-r) \alpha_{n_{j}} \beta_{n_{j}}^{\prime}}\right)\left\|x_{n_{j}}-p\right\|^{2}\right. \\
& \left.+\frac{1}{1-r}\left\langle f(p)-p, y_{n_{j}}-p\right\rangle-\frac{\left(1-\alpha_{n_{j}} r\right) \beta_{n_{j}}^{\prime \prime}}{2(1-r) \alpha_{n_{j}} \beta_{n_{j}}^{\prime}}\left\|e_{n_{j}}-p\right\|^{2}\right\} .
\end{aligned}
$$

Using (3.21) and (C2), we can conclude by Lemma 2.8 that $x_{n_{j}} \rightarrow p$ as $j \rightarrow \infty$. This is a contradiction and hence the sequence $\left\{x_{n}\right\}$ converges to $p \in \mathcal{F}$. Thus we completes the proof.

\section{Acknowledgements}

This work was supported by the Basic Science Research Program through the National Research Foundation(NRF) Grant funded by Ministry of Education of the republic of Korea(2015R1D1A1A09058177).

\section{References}

[1] Blum, E. and Oettli, W. (1994) From Optimization and Variational Inequality to Equilibrium Problems. Math. Stud., 63, 123-145.

[2] Kim, J.K. and Chang, S.S. (2014) Generalized Mixed Equilibrium Problems for an Infinite Family of Quasi- $\phi$-Nonexpansive Mappings in Banach Spaces. Nonlinear Anal. and Convex Anal., RIMS Kokyuroku, Kyoto Univ., 1923, 28-41.

[3] Yang, L. Zhao, F. and Kim, J.K. (2012) Hybrid Projection Method for Generalized 
Mixed Equilibrium Problem and Fixed Point Problem of Infinite Family of Asymptotically Quasi-Nonexpansive Mappings in Banach Spaces. Applied Mathematics and Computation, 218, 6072-6082. https://doi.org/10.1016/j.amc.2011.11.091

[4] Ahmad, M.K. and Salahuddin (2012) A Stable Perturbed Algorithms for a New Class of Generalized Nonlinear Implicit Quasi Variational Inclusions in Banach Spaces. Advances in Pure Mathematics, 2, 139-148. https://doi.org/10.4236/apm.2012.23021

[5] Ahmad, M.K., Salahuddin and Boulbrachene, M. (2008) Generalized Multivalued Vector Quasi Equlibrium Problems. Adv. Nonlinear Var. Inequal., 11, 25-34.

[6] Kim, J.K. (2011) Strong Convergence Theorems by Hybrid Projection Methods for Equilibrium Problems and Fixed Point Problems of the Asymptotically Quisi-Nonexpansive Mappings. Fixed Point Theory and Appl. https://doi.org/10.1186/1687-1812-2011-10

[7] Kim, J.K. (2015) Convergence Theorems of Iterative Sequences for Generalized Equilibrium Problems Involving Strictly Pseudocontractive Mappings in Hilbert Spaces. Jour. of Comput. Anal. and Appl., 18, 454-471.

[8] Kim, J.K., Cho, S.Y. and Qin, X.L. (2011) Some Results on Generalized Equilibrium Problems Involving Strictly Pseudocontractive Mappings. Acta Math. Sci., Series B, 31, 2041-2057,

[9] Kim, J.K., Cho, S.Y. and Qin, X.L. (2010) Hybrid Projection Algorithms for Generalized Equilibrium Problems and Strictly Pseudocontractive Mappings. Jour. of Inequalities and Appl., Article ID: 312062, 17 p.

[10] Kim, J.K. and Kim, K.S. (2010) New Systems of Generalized Mixed Variational Inequalities with Nonlinear Mappings in Hilbert Spaces. Jour. of Comput. Anal. and Appl., 12, 601-612.

[11] Kim, J.K. and Kim, D.S. (2004) A New System of Generalized Nonlinear Mixed Variational Inequalities in Hilbert Spaces. Jour. of Convex Analysis, 11, 235-243.

[12] Kim, J.K. and Salahuddin (2015) The Existence of Deterministic Random Generalized Vector Equilibrium Problems. Nonlinear Funct. Anal. Appl., 20, 453-464.

[13] Browder, F.E. and Petryshyn, W.V. (1967) Construction of Fixed Points of Nonlinear Mappings in Hilbert Spaces. J. Math. Anal. Appl., 20, 197-228. https://doi.org/10.1016/0022-247X(67)90085-6

[14] Halpern (1967) Fixed Points of Nonexpansive Maps. Bull. Amer. Math. Soc., 73, 957-961.

[15] Moudafi, A. (2000) Viscosity Approximation Methods for Fixed Point Problems. J. Math. Anal. Appl., 241, 46-55. https://doi.org/10.1006/jmaa.1999.6615

[16] Suzuki, T. (2007) Moudafi's Viscosity Approximations with Meir-Keeler Contractions. J. Math. Anal. Appl., 325, 342-352. https://doi.org/10.1016/j.jmaa.2006.01.080

[17] Petrusel, A. and Yao, J.C. (2008) Viscosity Approximation to Common Fixed Points of Families of Nonexpansive Mappings with Generalized Contractions Mappings. Nonlinear Anal. TMA, 69, 1100-1111. https://doi.org/10.1016/j.na.2007.06.016

[18] Takahashi, S. and Takahashi, W. (2007) Viscosity Approximation Methods for Equilibrium Problems and Fixed Point Problems in Hilbert Spaces. J. Math. Anal. Appl., 331, 506-515. https://doi.org/10.1016/j.jmaa.2006.08.036

[19] Ahmad, M.K. and Salahuddin (2006) Perturbed Three Step Approximation Process with Errors for a General Implicit Nonlinear Variational Inequalities. Int. J. Math. Math. Sci., Article ID: 43818. https://doi.org/10.1155/IJMMS/2006/43818

[20] Chen, R. and Song, Y. (2007) Convergence Common Fixed Point of Nonexpansive Semigroups. J. Comput. Appl. Math., 200, 566-575. 
https://doi.org/10.1016/j.cam.2006.01.009

[21] Liu, Z., Lee, J. and Kim, J.K. (2000) On Meir-Keeler Type Contractive Mappings with Diminishing Orbital Diameters. Nonlinear Funct. Anal. and Appl., 5, 73-83.

[22] Saeidi, S. (2009) Iterative Algorithms for Finding Common Solutions of Variational Inequalities and Systems of Equilibrium Problems and Fixed Points of Families and Semigroups of Nonexpansive Mappings. Nonlinear Anal. TMA, 70, 4195-4208. https://doi.org/10.1016/j.na.2008.09.009

[23] Suantai, S. and Cholamjiak, P. (2014) Algorithms for Solving Generalized Equilibrium Problems and Fixed Point of Nonexpansive Semigroups in Hilbert Spaces. Optimizations, 63, 799-815. https://doi.org/10.1080/02331934.2012.684355

[24] Takahashi, S. and Takahashi, W. (2008) Strong Convergence Theorem for a Generalized Equilibrium Problem and a Nonexpansive Mapping in Hilbert Spaces. Nonlinear Anal. TMA, 69, 1025-1023. https://doi.org/10.1016/j.na.2008.02.042

[25] Takahashi, W. and Toyoda, M. (2003) Weak Convergence Theorems for Nonexpansive Mappings and Monotone Mappings. J. Optim. Theory Appl., 118, 417-428. https://doi.org/10.1023/A:1025407607560

[26] Xu, H.K. (2005) A Strong Convergence Theorem for Contraction Semigroups in Banach Spaces. Bull. Austral Math. Soc., 72, 371-379. https://doi.org/10.1017/S000497270003519X

[27] Kim, J.K. and Tuyen, T.M. (2015) Viscosity Approximation Method with MeirKeeler Contractions for Common Zero of Accretive Operators in Banach Spaces. Fixed Point Theory and Appl., 2015.

[28] Kim, J.K. and Li, G. (2000) Nonlinear Ergodic Theorems for Reversible Semigroups of Lipschitzian Mappings in Banach Spaces. Dynamic Systems and Appl., 9, 255 268.

[29] Lau, A.T. (2008) Invariant Means and Fixed Point Properties of Semigroup of Nonexpansive Mappings. Taiwanese J. Math., 12, 1525-1542.

[30] Kim, J.K. and Li, G. (2000) Asymptotic Behavior for an Almost-Orbit of Nonexpansive Semigroups in Banach Spaces. Bull. Austral Math. Soc., 61, 345-350. https://doi.org/10.1017/S0004972700022358

[31] Lim, T.C. (2001) On Characterizations of Meir-Keeler Contractive Maps. Nonlinear Anal. TMA, 46, 113-120. https://doi.org/10.1016/S0362-546X(99)00448-4

[32] Takahashi, W. (1981) A Nonlinear Ergodic Theorem for an Amenable Semigroup of Nonexpansive Mappings in a Hilbert Spaces. Proc. Am. Math. Soc., 81, 253-256. https://doi.org/10.1090/S0002-9939-1981-0593468-X

[33] Li, G. and Kim, J.K. (2003) Nonlinear Ergodic Theorems for Commutative Semigroups of Non-Lipschitzian Mappings in Banach Spaces. Houston Jour. Math., 29, 231-246.

[34] Meir, A. and Keeler, E. (1969) A Theorem on Contractive Mappings. J. Math. Anal. Appl., 28, 326-329. https://doi.org/10.1016/0022-247X(69)90031-6

[35] Lin, Q. (2009) Viscosity Approximation for Common Fixed Points of a Nonexpansive Semigroup with a Generalized Contraction Mappings. Nonlinear Anal. TMA, 71, 5451-5457. https://doi.org/10.1016/j.na.2009.04.033

[36] Xu, H.K. (2002) Iterative Algorithms for Nonlinear Operators. J. London Math. Soc., 66, 240-256. https://doi.org/10.1112/S0024610702003332

[37] Suzuki, T. (2005) Strong Convergence of Krasnoselskic and Mann's Type Sequences for One Parameter Nonexpansive Semigroups without Brochner Integrals. J. Math. Anal. Appl., 305, 227-239. https://doi.org/10.1016/j.jmaa.2004.11.017 
[38] Zegeye, H. and Shahzad, N. (2012) Strong Convergence Theorem for a Common Fixed Solution of Variational Inequalities and Fixed Point Problems. Adv. Fixed Point Theory, 2, 374-397.

[39] Goebel, K. and Kirk, W.A. (1990) Topics in Metric Fixed Point Theory. Cambridge Studies Adv. Math., Vol. 28, Cambridge University Press, Cambridge, UK. https://doi.org/10.1017/CBO9780511526152

[40] Bruck, R.E. (1981) On the Convex Approximation Property and the Asymptotic Behaviour of Nonlinear Contractions in Banach Spaces. Israel J. Math., 38, 304-314. https://doi.org/10.1007/BF02762776

\section{Scientific Research Publishing}

Submit or recommend next manuscript to SCIRP and we will provide best service for you:

Accepting pre-submission inquiries through Email, Facebook, LinkedIn, Twitter, etc. A wide selection of journals (inclusive of 9 subjects, more than 200 journals) Providing 24-hour high-quality service User-friendly online submission system Fair and swift peer-review system Efficient typesetting and proofreading procedure Display of the result of downloads and visits, as well as the number of cited articles Maximum dissemination of your research work

Submit your manuscript at: http://papersubmission.scirp.org/ Or contact jamp@scirp.org 